\title{
Correction to: Co-crystals of 2,3-Dimethylquinoxaline (DMQ) and Dimethylglyoxime (DMG) in 1:1 and 1:2 Ratios and Hirshfeld Surface Analysis
}

\author{
Susobhan Biswas ${ }^{1} \cdot$ Rajat Saha $^{1} \cdot$ Ian M. Steele $^{2} \cdot$ Sanjay Kumar $^{1} \cdot$ Kamalendu Dey $^{3}$
}

Published online: 28 October 2017

(C) Springer Science+Business Media, LLC 2017

Correction to: J Chem Crystallogr (2013)

\section{3:493-501}

DOI 10.1007/s10870-013-0449-3

The original version of this article inadvertently contained errors in the CCDC numbers which have been quoted in the 'Electronic Supplementary Materials' section on page number 500 of the above article. The CCDC numbers 787144 and 787145 are not related to the crystal structures described in this paper. The correct CCDC numbers of compound I and II are 1571625 and 1571587.

The online version of the original article can be found under doi:10.1007/s10870-013-0449-3.

Sanjay Kumar

kumars@phys.jdvu.ac.in

Susobhan Biswas

susobhan1_b@yahoo.co.in

Kamalendu Dey

kdey_chem@rediffmail.com

1 Department of Physics, Jadavpur University, Jadavpur, Kolkata 700032, India

2 Department of Geophysical Sciences, The University of Chicago, Chicago, IL, USA

3 Department of Chemistry, University of Kalyani, Kalyani 741235, West Bengal, India 